\title{
Population pharmacokinetics of meropenem in febrile neutropenic patients in Korea.
}

\author{
Dong-Seok Yim ${ }^{1}$, Dong-Gun Lee, Su-Mi Choi, Hyon-Oh Lah ${ }^{1}$, Wan-Shik Shin \\ Departments of ${ }^{1}$ Pharmacology \& Internal Medicine
}

College of Medicine, The Catholic University of Korea, Seoul, Korea

\section{Introduction}

- Meropenem had been given as 1.5 gram/day ( 0.5 g every 8 hours) regimen, the lowest approved dose partially for healthcare cost containment in Korea without in-depth consideration of clinical efficacy

- Even in the case of critically ill patients i.e., neutropenia, this tendency for prescribing the 'minimum recommended dose' has not been altered.

- Therefore we carried out this study to explore the population PK of meropenem given as 0.5 gram every 8 hours in febrile neutropenic patients.

\section{Objectives}

To evaluate the "1.5 gram/day" regimen with pharmacometric tools

- Estimate population PK parameters

- Estimate a PD endpoint (Time above MIC, TAM) based upon MIC data from clinically isolated $P$.aeruginosa

\section{Subjects \& Methods}

1.Fifty seven neutropenic patients who were admitted to the hematologic malignancy unit at the Catholic Hematopoietic Stem Cell Transplantation (HSCT) center in Seoul, Korea.

2.Ethics Review and Consent

Written informed consent obtained in a form approved by the IRB of St. Mary's Hospital

\begin{tabular}{c|c}
\hline Characteristics & Median (Range) \\
\hline Age (y) & 36 (17-68) \\
Number & 57 (female 27/ male 30) \\
Height (cm) & $162.6(141-190)$ \\
Weight (kg) & 61.4 (45-95.8) \\
Underlying Disease* & AML 36; ALL 14; MDS 4; others 4 \\
CL $L_{\sigma_{t} \text { (m/minn) }}$ & 121 (26.4152.2) \\
\hline
\end{tabular}

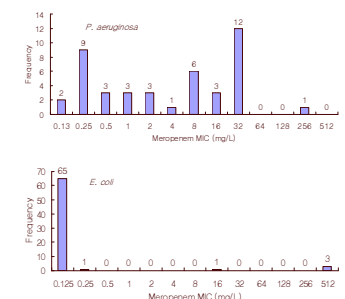

3. MIC's of clinically isolated $P$. aeruginosa and $t$. coll strains, which were from neutropenic patients from the same unit from 2000 to 2003, were used to estimate the TAM.

4. Meropenem administration and Blood sampling

Dosage regimen $-0.5 \mathrm{~g}$ of meropenem (10 min infusion) every 8 hours

Venous blood withdrawn 2-3 $\mathrm{h}$ (for peak) and 5-6 $\mathrm{h}$ (for trough) after the injection at steady state.

5. Plasma Meropenem Assay

HPLC (based on the method reported by Ip et al., 1998)

- LOQ $0.5 \mathrm{mg} / \mathrm{L}$

- Linear from 0.5 to $50 \mathrm{mg} / \mathrm{L}$ of standard solution.

6. Population PK model development : NONMEM (version 5.1.1).

- PREDPP subroutines ADVAN1 TRANS2 used

- Structural model : 1-compartment, first order elimination

$\mathrm{CL}_{\mathrm{i}}=\mathrm{CL}_{\text {pop }} \times \mathrm{e}^{\eta \mathrm{CL}}, \mathrm{Vd}_{\mathrm{i}}=\mathrm{Vd}_{\text {pop }} \times \mathrm{e}^{\eta \mathrm{Vd}}$

$\eta_{\mathrm{CL}}$ and $\eta_{\mathrm{Vd}}$ : independent random-error variables (means 0 and variance of $\omega_{C L}^{2}$ and $\omega_{V d}^{2}$, respectively)

- Residual error: $\mathrm{C}_{\mathrm{ij}}=\mathrm{C}_{\mathrm{ij}(\text { pred })}\left(1+\varepsilon_{\text {propij }}\right)$

$C_{i i j}$ : observed jth concentration in the ith individual;

$\mathrm{C}_{\text {i }}$ : the concentration predicted for the ith individual

- FOCE method with interaction used

- Bootstrap method: More than 1,000 successfully minimized re-samples to

obtain $95 \%$ confidence intervals $(\mathrm{Cl})$ for parameter estimates

7. Simulation of Concentrations

- A Monte-Carlo clinical trial simulation experiment using Pharsight Trial

Simulator ${ }^{\circledR}$ (Pharsight Corporation, version 2.1.2, Mountain View, CA, USA)

- MICs : simple discrete proportions, just as observed in the MIC test

histograms, were used instead of assuming any distribution model.

- a total of 1,000 virtual patients' data were generated for differing dosage regimens and pathogens.

- Simulated TAM (Time above MIC) : assumption of $92 \%$ of total concentration unbound (Dreetz et al., 1996)

\section{Results}

Population PK

\begin{tabular}{|c|c|c|c|}
\hline Parameter & Meaning & Symbol & $\begin{array}{l}\text { Mean and 95\% } \\
\text { C.I. }{ }^{\pi}\end{array}$ \\
\hline \multirow{2}{*}{$\mathrm{CL}(\mathrm{L} / \mathrm{h})$} & \multirow{2}{*}{$\begin{array}{c}C_{\text {pop }}=\theta_{1} \times\left(C L_{\sigma_{r}}{ }^{+1 / 120)}\right. \\
C L_{i}=C L_{p o p} \times e^{n C L}\end{array}$} & $\theta_{1}$ & $9.7(7.56-11.82)$ \\
\hline & & $\omega_{\mathrm{CL}}$ & $0.73(0.66-0.79)$ \\
\hline \multirow{2}{*}{ Vd (L) } & \multirow{2}{*}{$\begin{array}{c}V_{\text {pop }}=\theta_{2} X\left(\text { Body weight }{ }^{\ddagger} / 61\right) \\
V d_{i}=V d_{p o p} \times e^{n V d}\end{array}$} & $\Theta_{2}$ & $14.6(11.08-18.1)$ \\
\hline & & $\omega_{\text {vd }}$ & $0.54(0.53-0.86)$ \\
\hline Correlation & $\begin{array}{l}\text { Correlation coefficient } \\
\text { between (CL and Vd) }\end{array}$ & - & $0.998(0.88-1)$ \\
\hline $\begin{array}{c}\begin{array}{c}\text { Half-life } \\
\text { (hr) }\end{array} \\
\end{array}$ & $t_{1 / 2}{ }^{*}$ & - & $1.04(0.94-1.14)$ \\
\hline $\begin{array}{l}\text { Residual } \\
\text { Error }\end{array}$ & $\begin{array}{c}\left.C_{i j} \text { (Observed Conc.) }\right)= \\
C_{\text {iipreses) }} \times\left(1+\varepsilon_{\text {prop }}\right)\end{array}$ & $\sigma_{\text {prop }}$ & $0.298(0.00-0.31)$ \\
\hline
\end{tabular}

Simulated TAM's
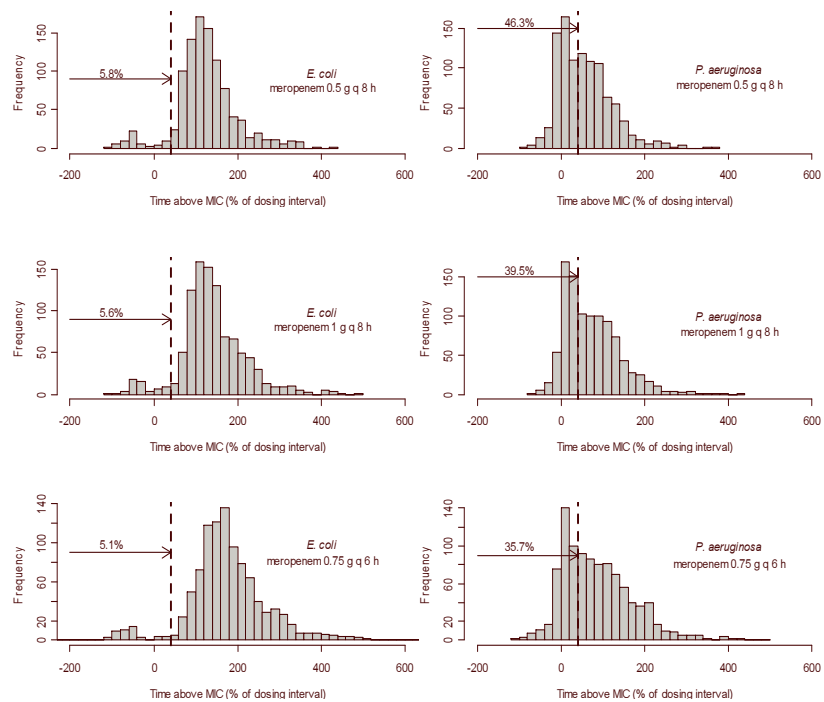

Frequency distribution of TAM (Time above MIC) as percentage of the dosing interval) for $P$. aeruginosa and $E$. coli isolates when meropenem was given $0.5 \mathrm{~g} \mathrm{q} 8 \mathrm{~h}, 1 \mathrm{~g} \mathrm{q} 8 \mathrm{~h}$ and $0.75 \mathrm{~g} \mathrm{q} 6 \mathrm{~h}$ in 1000 simulated patients. A TAM greater than $40 \%$ of the dosing interval was chosen as a cut-off point for clinical efficacy. Dotted lines indicate $40 \%$ of the dosing interval and percentage values above the arrows indicate the proportion of patients with TAM shorter than $40 \%$ of the dosing interval.

\section{Discussion}

Meropenem $1 \mathrm{~g} \mathrm{q} 8 \mathrm{~h}$ in neutropenic Korean patients is expected to be more effective than a $0.5 \mathrm{~g} \mathrm{q} 8 \mathrm{~h}$ regimen which was implemented without pharmacodynamic consideration of highly resistant strains.

For $\mathrm{P}$. aeruginosa, we need a comprehensive re-evaluation of treatment strategies including aminoglycoside combination, meropenem monotherapy with higher dosage or consideration of other susceptible antibiotics.

\section{References}

Ip M, Au C, Cheung SW, Chan CY, Cheng AF. A rapid high-performance liquid chromatographic assay for cefepime, cefpirome and meropenem. J Antimicrob Chemother 1998; 42:121-3

Dreetz M, Hamacher J, Eller J, et al. Serum bactericidal activities and comparative pharmacokinetics of meropenem and imipenem-cilastatin. Antimicrob Agents Chemother 1996; 40:105-9.

$$
T A M=\frac{\ln \left(\frac{C_{\text {SSpeak }}}{M C}\right)}{\frac{C L}{V d}}
$$

